\title{
Research of Global Features of 3D Color Histogram with Similarity and Dissimilarity Metrics for Image Processing
}

\author{
A. Jeya Christy, K. Dhanalakshmi
}

\begin{abstract}
A review of the global feature color comprising of seven different metrics are discussed here. The color is the most powerful feature for describing the images. This study contains four sections. The first section explains the related techniques used in various papers. The second section explains the two different kinds of metrics. (1) Similarity metrics such as Cosine and Correlation and (2) Dissimilarity metrics such as Euclidean, Manhattan, Bhattacharyya, Chi-Squared and Intersection. The third section explains experiment results using CALTECHUCSD Birds-200 image library. The fourth section gives the conclusion and future work. In this experiment, the query image can be divided into trained (indexed) or untrained (non-indexed). In the similarity metric analysis, the experimental results show that the cosine similarity gives better similarity score than correlation. Similarly, in the dissimilarity metric analysis, the Bhattacharyya gives a better result than other distance metrics.
\end{abstract}

Key Words: Color histogram, Similarity metric, Dissimilarity metric, Cosine similarity, Correlation Euclidean, Manhattan, Bhattacharyya, Chi-Squared and Intersection

\section{INTRODUCTION}

Image Retrieval is the process of getting the output as image depends upon the query given by the user. The query may be text or image. If the query is text then it used Text Based Image retrieval (TBIR).If the query is image then it used Content Based Image Retrieval(CBIR).

Text Based Image Retrieval has two major drawbacks. First, it needs manual image annotation. So it leads to time consuming and therefore costly. Second, human annotation leads to typos and ambiguous problems. In addition, some images could not be annotated due to difficulty in describing the image content in words.

Content Based Image Retrieval is an approach in digital image processing. CBIR is used to search the image through an image database and finds out the most relevant or similar ones to the input query image by means of these visual contents (features) such as color [1], shape[2], texture[3], appearance pattern or any combination of them [4-6].

The CBIR system extracts the features from images in the database which is based on the value of the image pixels. These features are stored in a feature database which is represented by the compact form of a fixed length of real valued multi component feature vectors [38].

Google uses Reverse image search by means of allowing users to upload an image and then search for the matched and similar images.

Revised Manuscript Received on January 2, 2020.

A. Jeya Christy, Research Scholar, Department of Computer Science and Engineering, Vaigai College of Engineering, Madurai, Tamil Nadu, India.

Dr. K. Dhanalakshmi, Professor, Department of Computer Science and Engineering, PSNA College of Engineering and Technology, Dindigul, Tamil Nadu, India.
In CBIR, RGB color space where the gray level intensity is represented as the sum of red, green and blue.

Vellaikal.A,JayKuo.C.C and Dao.S [33] explained that RGB Color space is not perceptually uniform. Here all RGB components have equal importance so those values have to be quantized with the same precision.This problem can be handled by using a weight matrix during distance measure[34]

Common color features such as color-covariance matrix, color histogram, color moments and color coherence vector.

The uses of color has been experimented in color matching[25], similarity searching [26][27], region searching [28][29], target searching [30][31], and semantic searching[32].

In these visual content, the color feature is the most powerful approach because which is automatically extracted from the image.

The color histogram is a stable object representation which is used to differentiate among a large number of objects. Swain and Ballard[7] have demonstrated the use of color image indexing and in order to solve the object identification problem, they introduced a fast version of Histogram intersection.

Ahmed J. Afifi and Wesam M. Ashour [39] Proposed color based retrieval system for comparing the similarity between the two images in order to handle the rotation and translation of an image.

John R. Smith and Shih-Fu Chang [24] Presented Visual SEEK system which is a hybrid system that utilized a salient image region and their color, sizes, spatial location and relationships in order to compare images. The strategies utilized by this system for computing the complex queries and presented some preliminary results that indicate the system's efficiency and power.

3D Color histogram means that partitioning the color space into a set of $3 \mathrm{D}$ bins and implements an $8 * 8 * 8$ color histogram.

Ji-Hee Han, Sejung Yang, and Byung-Uk Lee [13] proposed a new 3-D color histogram method to obtain the uniform distribution in the intensity domain.

Funt and Finlayson [8] presented the color constant color indexing method to extend [7] color indexing method to be illumination constant locally.

Ju Han and Kai-kuang Ma [9] presented a new approach Fuzzy Color Histogram (FCH) by considering the color similarity of each pixel's color correlated to all the histogram bins through fuzzy set membership function. Hence it proficiently addresses the noisy interference and quantization errors. 
k a-Man Wong, Chun-Ho Cheung and Lai-Man P.O[10] proposed a novel Merged Color Histogram (MCH) to generate a common color palette before the use of histogram intersection and similarity measurement. The common color palette is generated by using CQ (color Quantization), Color space CIEluv transformation, intra and inter palette merging. Hence, the MCH method achieved precision 11-32\% more relevant in the first 20 retrievals and recall about $14-23 \%$ more relevant images in the first 100 retrievals when compared to the Conventional RGB based histogram method.

Stricker.M and Dimai.A [40][41] proposed two approaches in order to achieve the efficiency of the system. (1)They computed the first three moments of the histograms of each color channels and used it as an index. (2)The image is represented only by average and covariance matrices of color distribution.

\section{SIMILARITY / DISSIMILARITY METRICS}

A metric is a tool for measuring similarity and dissimilarity between two objects/ images. In order to find the similarity between the images using the following metrics such as correlation coefficient metric, cosine similarity whereas to find the dissimilarity between the images using the distance metrics such as Euclidean, Manhattan(City Block), Bhattacharyya, Chi-Squared and Intersection.

\section{a) Euclidean distance}

It is simple and also most commonly used in color based image retrieval. It is used to measure the distance between two objects in space [12][36].In the Euclidean distance, Pythagorean theorem gives the distance between two points. In Cartesian coordinates, if $a=(a 1, a 2, a 3)$ and $b=(b 1, b 2$, b3) are two points in this Euclidean space. Euclidean distance between two points ' $a$ ' \& ' $b$ ' are computed using the following equation.

$d(a, b)=\sqrt{\left(a_{1}-b_{1}\right)^{2}+\left(a_{2}-b_{2}\right)^{2}+\left(a_{3}-b_{3}\right)^{2}}$

Ivan Dokmanic, Reza Parhizkar, JuriRanieri, and Martin Vetterli [11] have concluded the Euclidean metric can be used by solving a labeling problem.

Archana Singh, AvantikaYadav and Ajay Rana [14] have implemented k-means using Euclidean distance metric for the 2D histogram. It gave a better result than using Manhattan.

\section{b) Manhattan distance}

It is a distance between the two points is the sum of the absolute differences of their Cartesian coordinates (a,b). If $\mathrm{a}=(\mathrm{a} 1, \mathrm{a} 2) \& \mathrm{~b}=(\mathrm{b} 1, \mathrm{~b} 2)$ are two points in a plane $\mathrm{ab}$.

$$
\begin{aligned}
\mathrm{d}(a, b) & =\sum_{i=1}^{n}\left|a_{i}-b_{i}\right| \\
& =\left|a_{1}-b_{1}\right|+\left|a_{2}-b_{2}\right|
\end{aligned}
$$

M. D. Malkauthekar [15] presented the face expression recognition problem using Manhattan distance \& Euclidean distance. He mentioned that the Euclidean method does not consider the variability of values in all dimensions particularly for images with changed angle. In which, Manhattan distance gave a better result for recognizing the images.

\section{c) Cosine similarity}

It measures the similarity between non-zero vectors in product space. In order to determine cosine similarity, it finds the cosine of the angle between the two objects but not magnitude. Here two vector attributes a \& b, the cosine similarity, $\cos \theta(\theta)$ is represented using the dot product and magnitude as follows.

$$
d(a, b)=\cos (\theta)=\frac{a \cdot b}{\|a\|\|b\|}
$$

In this how similar two images are the same in the angle instead of scaling. If the angle $\theta$ is 1 means both images are in same direction otherwise they are in different directions.

The cosine similarity metric is commonly applied in the discriminant analysis in pattern recognition. This metric is suffered from the distance problem and the angle problem that deteriorate the classification performance. Hence Chengjun Liu [16] proposed a new similarity measure to overcome the above problems by integrating the absolute value of the angular measure.

\section{d) Bhattacharyya distance}

It measures the similarity of two discrete or continuous probability distribution in statistics. It is related to the Bhattacharyya coefficient, which is used to determine the relative closeness of the two samples. For probability distribution $a$ and $b$ over the same domain $X$, the Bhattacharyya distance is calculated using the following formula

$$
B C(a, b)=\sum_{x \in X} \sqrt{a(x) b(x)}
$$

For continuous probability function,

$$
B C(a, b)=\int \sqrt{a(x) b(x) d x}
$$

For both cases $0 \leq \mathrm{BC}(\mathrm{a}, \mathrm{b}) \leq 1$ and $0 \leq \mathrm{d} \leq \infty$

The Skew Bhattacharyya distance is often used in machine learning, statistical signal processing and information fusion [17][18][19]. A triparametric generalization of the Bhattacharyya distance has been reported by Frank Nielsen [20].

\section{e) Chi-Squared distance}

It is used for comparing two histograms (the vector with non-negative values) which are calculated using the following formula

$$
\chi^{2}(a, b)=\frac{1}{2} \sum_{i=1}^{n} \frac{\left(a_{i}-b_{i}\right)^{2}}{\left(a_{i}+b_{i}\right)}
$$

The correspondence problem is particularly complicated for 3D biomedical model surfaces, especially for roundish and approximate symmetric organs such as stomach, liver, kidney etc. Jun Feng and Horace H.S. Ip [21] applied ChiSquare Goodness-of-Fit test to establish 3D point correspondence.

\section{f) Correlation distance}

It is used to measure the relationship between the two histograms. Correlation function returns the value between-1 and 1 in which it returns 1 means a positive relationship,-1 means negative relationship \& 0 means no relationship. The Correlation coefficient $\left(\mathrm{C}_{\mathrm{c}}\right)$ is computed using the following formula[35] 


$$
C_{c}=\frac{n\left(\sum a b\right)-\left(\sum a\right)\left(\sum b\right)}{\sqrt{\left[n \sum a^{2}-\left(\sum a\right)^{2}\right]\left[n \sum b^{2}-\left(\sum b\right)^{2}\right]}}
$$

Chengjun Liu [16] presented the normalized correlation to overcome the angle problem of the cosine similarity.

Siddhartha Bhattacharyya, ParamarthaDutta, UjjwalMaulik and Prashanta Kumar Nandi [35] Presented a parallel neural network architecture for segmentation of true color images. They used the correlation coefficient between the original true color images and segmented outputs.

\section{g) Intersection}

it is used to find the similarity of two histograms(discretized probability distribution) namely $H_{I} \& H_{2}$ In programming aspect, the correlation and intersection methods need sorting in reverse order

$$
d\left(H_{1}, H_{2}\right)=\sum_{i} \min \left(H_{I}(i), H_{2}(i)\right)
$$

Swain \& Ballard [7] have demonstrated the histogram intersection is able to eliminate the influence of color contributed from the background pixels.

\section{EXPERIMENT RESULTS}

\section{A) Build 3D RGB Color Histogram:}

In figure 1 , the $\mathrm{x}$-axis represents the pixel color values (Bins) and the $y$-axis represents the number of pixels.
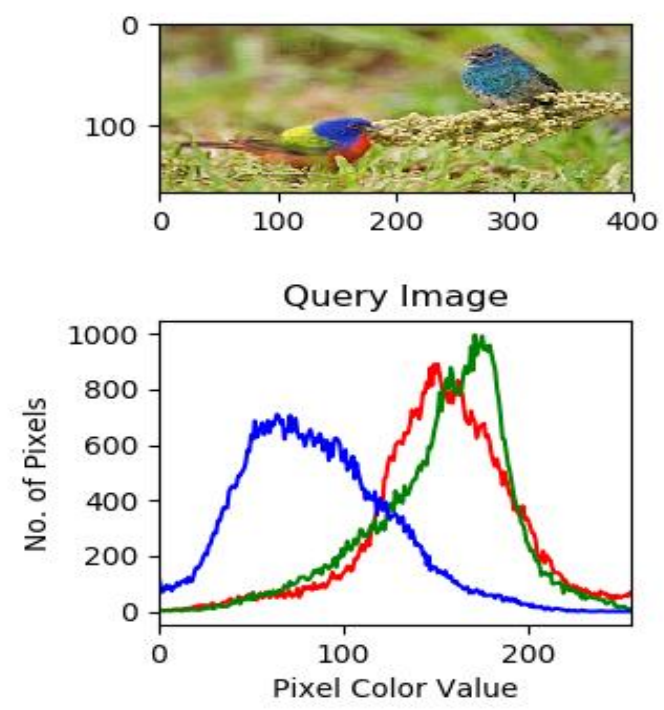

Figure 1: Histogram for Query image

In the experiment, 105 random PNG (Portable Network Graphics) images in the CALTECH-UCSD Birds-200 image library were adopted, which was divided into 7 equal size classes (Rufous humming, yellow breasted, painted bunting, Laysan Albatross, Indigo Bunting, Green Violetear\& Crested Auklet), and 15 images in each. The images size of height 166, width 400 and 3 channels (Red, Green, Blue). A python programming experiment was used. In this implementation, the 3D RGB Color histogram reshaped every image to $512(=8 * 8 * 8)$ because each Channel $(\mathrm{R}, \mathrm{G} \&$ B) used 8 bins. It shows that all the 7 distance metrics could correctly retrieve the relevant images. Commonly people pay more attention to the first ten retrieved results. So this paper reported the accuracy of the first ten results.

In the experiments, relevant images are retrieved using seven different similarity/dissimilarity metrics with respect to the query image given by the user. In addition to this, the query image is taken externally (untrained image) and also internally (trained image) for testing.

In this, the image retrieval system primarily having 4 steps

1. Define features: The most powerful low- level visual feature color was used which is automatically extracted from the image dataset

In order to compute the 3D histogram with 8 bins, it normalizes the histogram in terms of pixel counts. Here pixel count described in real valued percentages instead of a raw integer.

2. Quantifying and indexing: Indexing images in the image dataset based on step 1 that means extract a 3D RGB histogram from each image, store its feature in a dictionary and write it to the file.

3. Define Similarity metric: The various metrics such as Euclidean, Cosine, etc., are defined to measure similarity/dissimilarity between images.

4. Searching

4.1 The retrieval system allows the user to give the query image. The query image can be categorized into trained (internal) and Untrained (External).

4.2 Now the image retrieval system measures the distance between the query image and indexed images in the image dataset.

All the metrics (except Intersection method) work in accordance with the following property.

IF the distance between the two feature vectors (query image \& Indexed image) is Zero/One THEN

It is the perfect similarity.

ELSE IF the distance between two feature vectors of the images smaller THEN

It is very close to the query.

ELSE (Larger)

It may be far from the query image or it is irrelevant ENDIF

The intersection Method works in accordance with the following property.

IF the distance between the two feature vectors (query image \& Indexed image) is larger THEN

It is the perfect similarity or close to the query.

ELSE (Smallest)

It may be far from the query image or it is irrelevant. ENDIF

4.3 Depends on the metrics, the system is returned the relevant images to the user.

4.3.1 The relevant images are retrieved using a ChiSquared distance metric (Customized) is shown in Figure 2. In which, the Query image is a trained image (Internal Image).

It is mainly used to compare the histograms. Hence the distance between the query image and Topmost image is 0.000 i.e., exact image. Likewise, all the metrics are used for this query and obtain the same result for the topmost retrieved image.

4.3.2 The relevant images are retrieved using a ChiSquared distance metric (Customized) is shown in Figure 3. In which, the query image is an unknown image (external image) to the image dataset. 
In spite of this, the distance between the query and the first image in the result is 1.705 .

4.3.3 The retrieved relevant images using Euclidean distance are shown in Figure 4. It is a very familiar method. To obtain the most relevant images, the retrieval system is developed to sort the distance score because the smaller value indicates a higher similarity. Hence the more relevant images are at the front of the resultant list.

4.3.4 The result retrieved using the Manhattan distance metric is shown in Figure 5. The distance is calculated using 4 connected neighborhood. In the implementation, the results should be sorted because smaller value indicates a higher similarity between the images.

4.3.5 The relevant images are retrieved using Bhattacharyya distance is shown in Figure 6. In which, the distance score needs to be sorted in the implementation. So, the distance between the query image and the topmost image returns 0.493 . It gives a better result when compared to other distance metrics.

4.3.6 The relevant images retrieved using Correlation method is shown in Figure 7. In order to get the result, the distance values need to be sorted in descending order or reverse. Here the Similarity measure between a query image and a topmost image is 0.696. For perfect matching, it returns the similarity value is 1 .

4.3.7 The relevant images retrieved using Intersection Method is shown in Figure 8. For this, the result should sort in reverse. So, the distance between the query image and the topmost image returns 2.671 .

4.3.8 The relevant images retrieved using Cosine Similarity is shown in Figure 9. In order to get more relevant images at the top, the result should sort in reverse in the programming. It gives a better result than the other Similarity measures. For the Perfect matched image, this similarity function returns the value is 1 .

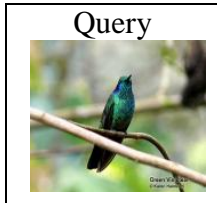

1.406

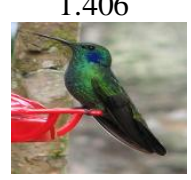

1.748

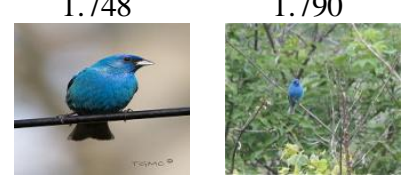

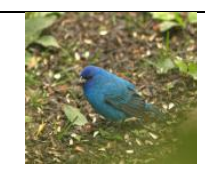
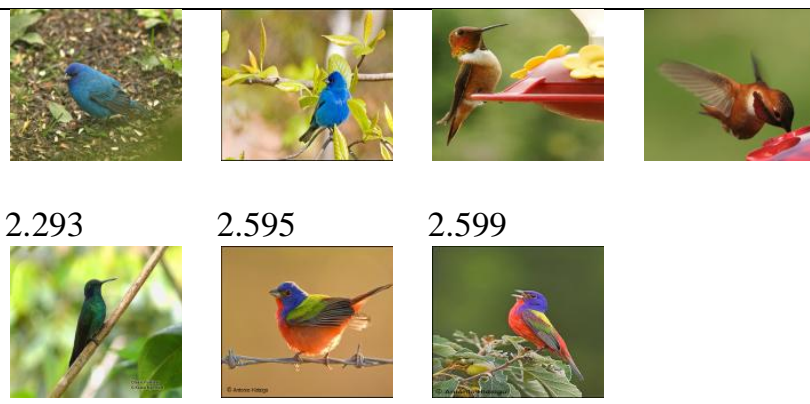

Figure 3: query image as an external image using chisquared distance metric (customized)
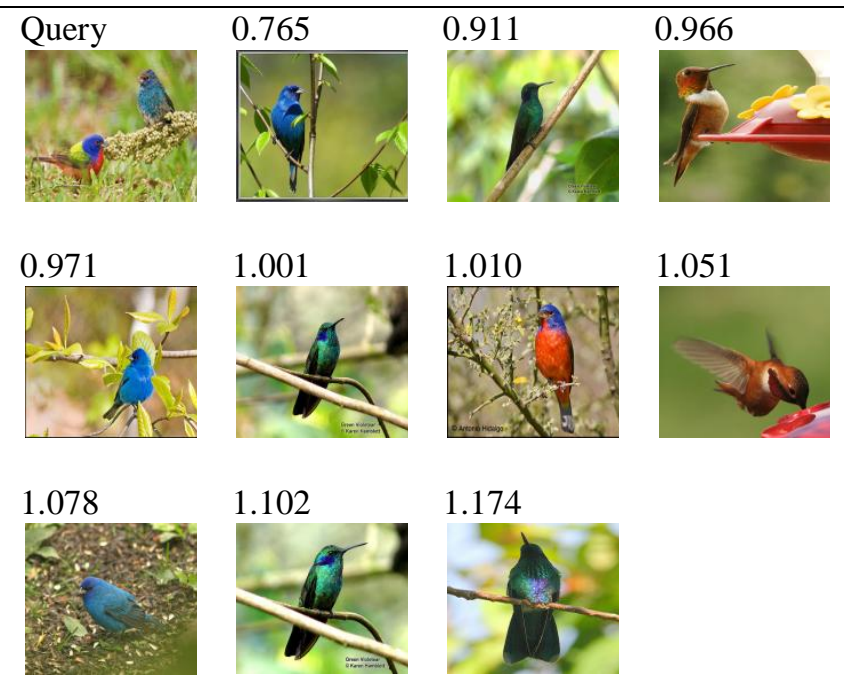

Figure 4: Euclidean distance
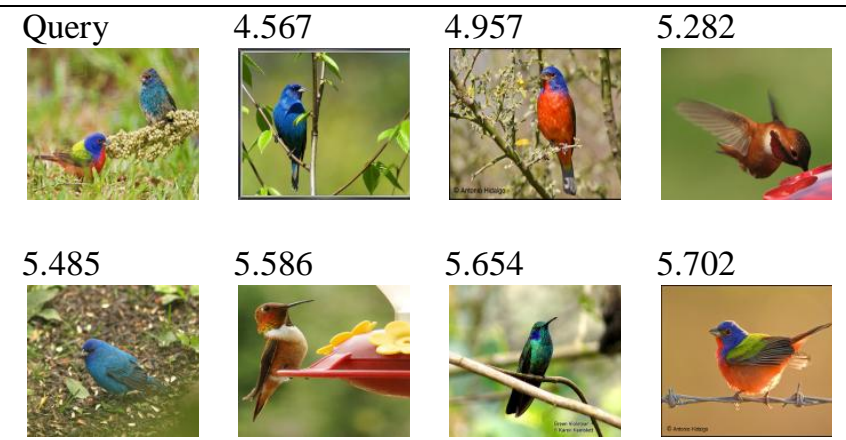

5.742
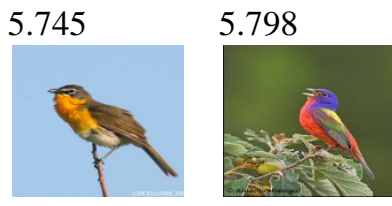

Figure 5: Manhattan distance metric

Figure 2: Query image as an internal image Using chisquared distance metric (customized)

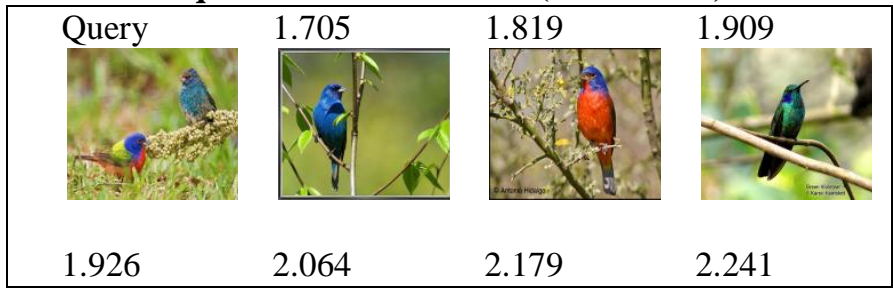

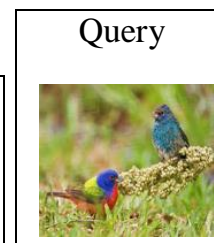

0.493

0.511

0.514
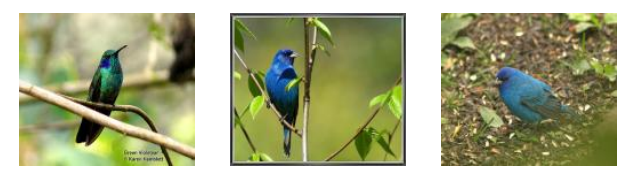

0.523

0.535

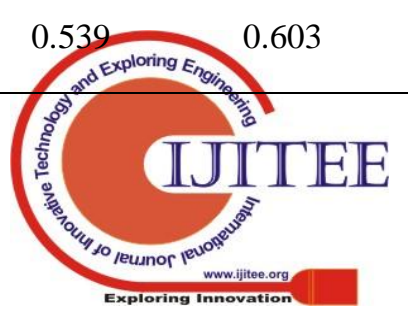




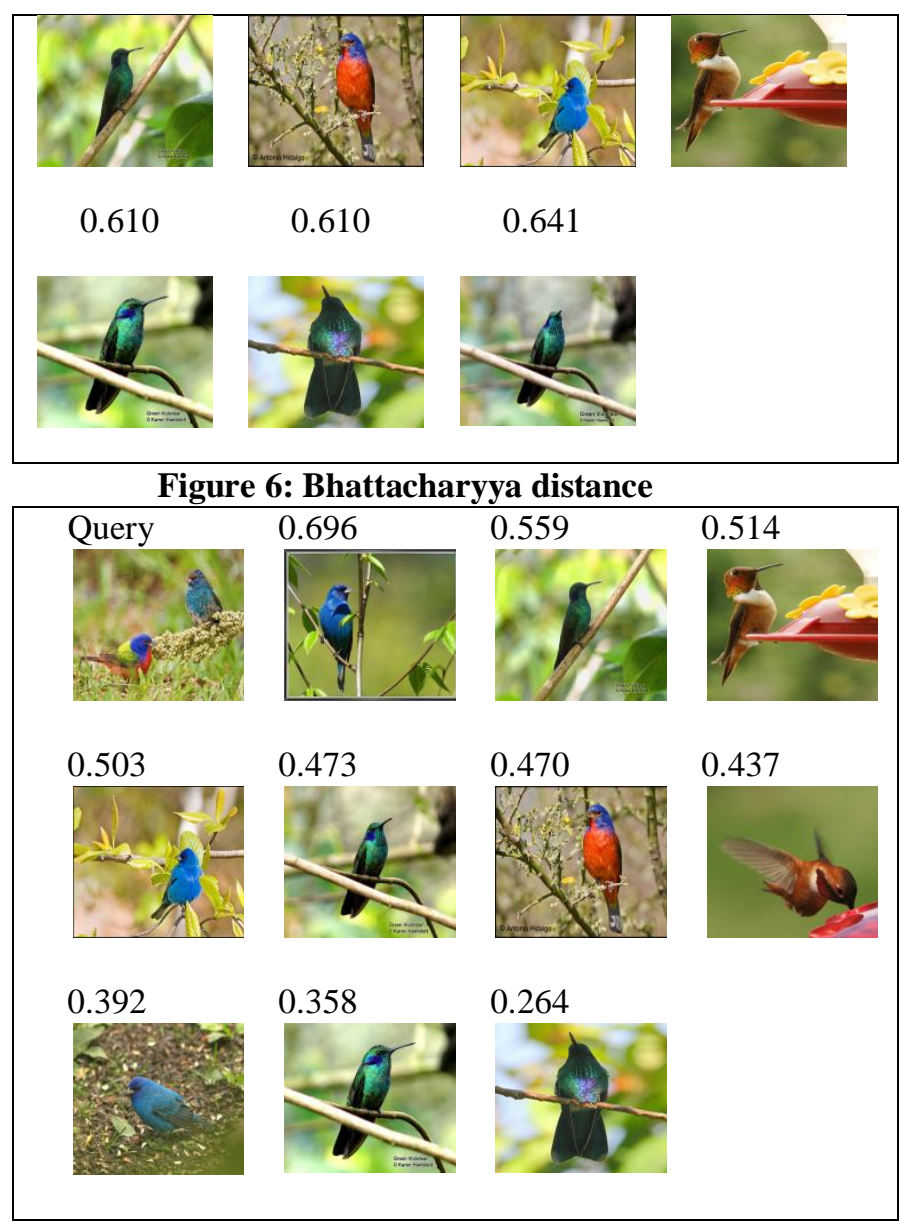

Figure 7: Correlation method

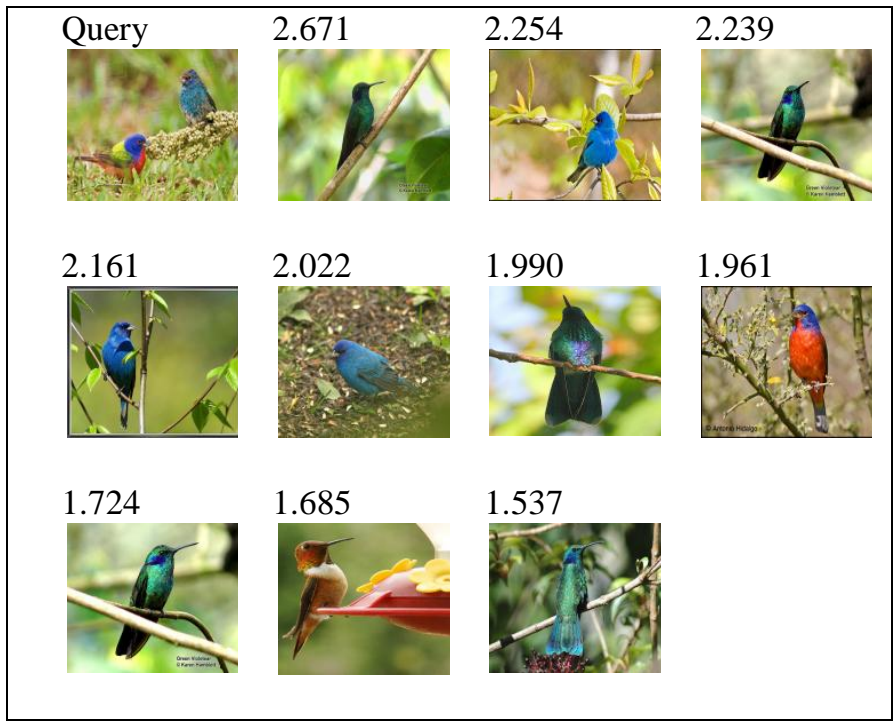

Figure 8: Intersection Method

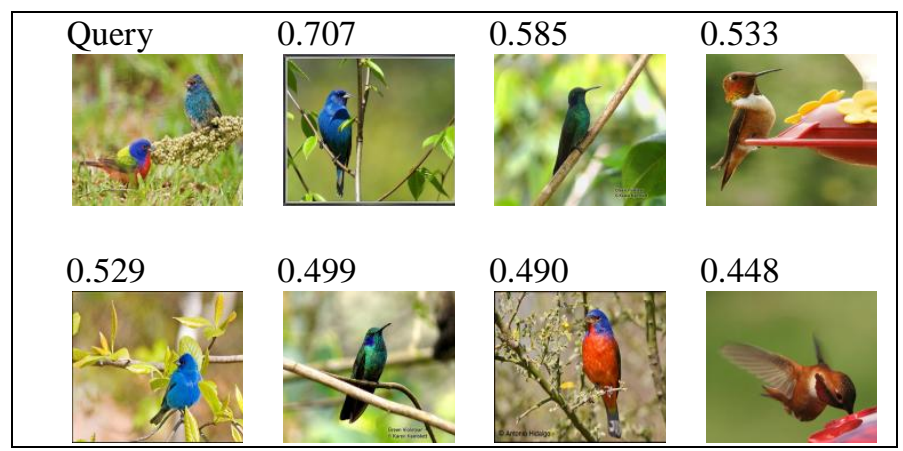

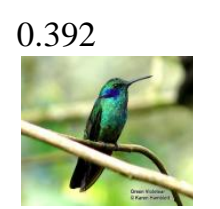

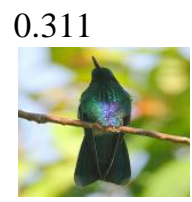

Figure 9: Cosine Similarity

The perfect matched image(exact image) means the query image is trained by the system which can be one of the images in the image dataset. This paper obtained the result of the perfect image associated with the query image can be defined in three ways

Chi-Squared, Bhattacharyya, Euclidean and Manhattan distance metrics return 0 for the perfect matched image.

Correlation and Cosine similarity metrics return 1 for the perfect matched image.

Intersection returns a larger value for the perfect matched image.

\section{B) Retrieval Efficiency}

It is measured by the performance in terms of precision, recall and accuracy. In order to determine the retrieval efficiency, the standard formulas have been used[22][23].

Precision: It is used to find how many irrelevant images did we given to the user[37]. Precision is calculated by using the following formula,

$$
\text { Precision }=\frac{\mathrm{TP}}{\mathrm{TP}+\mathrm{FP}}=\frac{\begin{array}{c}
\text { Number of relevant } \\
\text { images retrieved }
\end{array}}{\begin{array}{c}
\text { Total Number of } \\
\text { images returned }
\end{array}}
$$

Where TP- True Positive: denotes how many relevant images are retrieved?

FP- False Negative: denotes how many non relevant images are retrieved?

Recall: It is used to find how many relevant images we missed to give to the user[37].

$$
\text { Recall }=\frac{\mathrm{TP}}{\mathrm{TP}+\mathrm{FN}}=\frac{\begin{array}{c}
\text { No. of relevant images } \\
\text { retrieved }
\end{array}}{\begin{array}{c}
\text { Total No. of relevant images } \\
\text { in the image database }
\end{array}}
$$

Where FN- False Negative: denotes how many relevant images are not retrieved? and recall.

Accuracy: It is calculated with the help of precision

$$
\text { Accuracy }=\frac{(\text { Precision }+ \text { Recall })}{2}
$$

High precision indicates that less irrelevant images are retrieved or more relevant images are retrieved in the result, while high recall indicates that less relevant images are missed. 


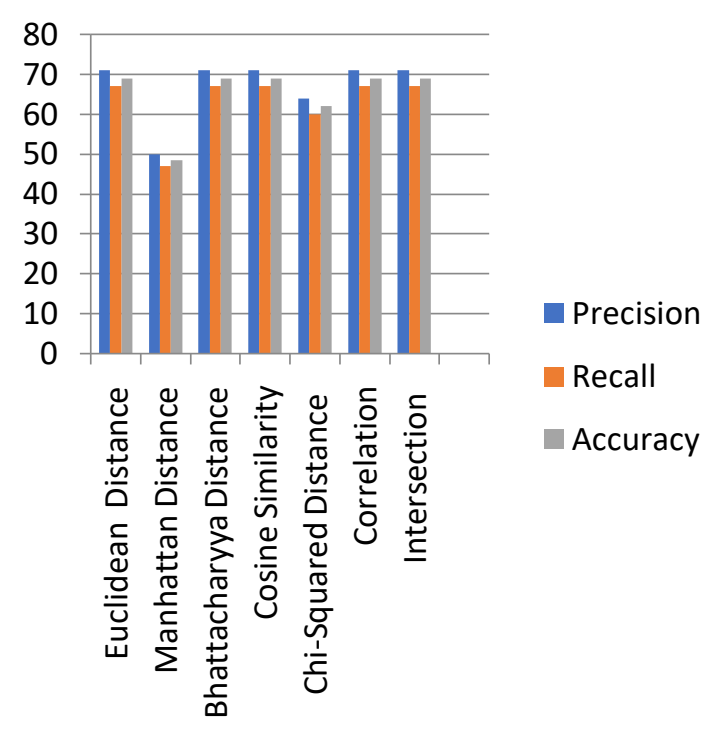

Figure 11: Comparison of various distance metrics with performance measures.

\section{CONCLUSION}

This paper has shown the performance of 3D Color histogram in RGB Color space with the help of distance metrics (dissimilarity) such as Euclidean, Manhattan, Bhattacharyya, Chi-Squared and Intersection could accurately retrieve the similar and matched images relevant to the query image using CALTECH-UCSD Birds-200 image library.

In addition to that, the similarity metrics such as Cosine and Correlation were applied on the image dataset. In the similarity metric analysis, the cosine similarity gives better similarity score than correlation. Similarly, in the dissimilarity metric analysis, the Bhattacharyya gives a better result than other distance metrics. Future work involves running this experiment on a larger dataset.

\section{REFERENCES}

1. J. Smith, S.-F. Chang, "Tools And Techniques for Color Image Retrieval", In Symposium on Electronic Imaging: Science and Technology - Storage \& Retrieval for Image and Video Databases IV, volume 2670, San Jose, CA, February 1996. IS\&T/SPIE.

2. D. Dimov, "Fast, Shape Based Image Retrieval", In Proceedings of International Conference on Computer Systems and Technologies, CompSysTech'2003.

3. J. Smith, S.-F. Chang, "Transform Features for Texture Classification and Discrimination in Large Image Databases", In Proceedings of the I.E.E.E. International Conference on Image Processing (ICIP-94), vol. 3, pp. 407-411, November 1994.

4. J.wang and G.Wiederhold, "SIMPLIcity:Semantics-Sensitive Integrated Matching for Picture Libraries", IEEE transaction on Pattern Analysis and Machine Intelligence" vol.23 no. 9, pp. 1-17, September 2001.

5. Y.Rui,T.Huang and S.F.Chang, "Image Retrieval:Curren Techniques, Promising Directions and Open Issues", Journal of Visual Communication and Image Representation, Vol. 10,pp.3962, March,1999.

6. M.Flicker, et al., "Query by Image and Video Content: The QBIC system”, IEEE Computer, pp. 23-32, vol.28, no.9, September 1995.

7. M.Swain and D.Ballard "Color Indexing", International journal of Computer Vision, Vol.7 no.1, pp.11-32, 1991.

8. B.V.Funt and G.D.Finlayson, "Color Constant Color Indexing", Pattern Analysis Machine Intelligence, Vol. 17, no. 5, pp.522529,1995

9. Ju Han and Kai-Kuang Ma, "Fuzzy Color Histogram and its use in Color Image Retrieval “, IEEE Transaction on Image Processing, Vol. 11, No. 8,August 2002.
10. k a-Man Wong, Chun-Ho Cheung and Lai-Man P.O, "MergedColor Histogram for Color Image Retrieval" , In Proceedings of the IEEE International Conference on Image Processing, Vol. 3, pp.949-952, 2002.

11. Ivan Dokmanic, Reza Parhizkar, JuriRanieri, and Martin Vetterli, "Euclidean Distance Matrices: Essential theory, Algorithms, and Applications", IEEE Signal Processing Magazine, Vol. 32, pp. 12-30, Issue: 6, Nov. 2015

12. L. D. Chase, " Euclidean Distance", College of Natural Resources, Colorado State University, Fort Collins, Colorado, USA, 824-146294, NR 505, December 8, 2008.

13. Ji-Hee Han, Sejung Yang, and Byung-Uk Lee, ”A Novel 3-D Color Histogram Equalization Method With Uniform 1-D Gray Scale Histogram “, IEEE Transactions On Image Processing, Vol. 20, pp. 506-512, issue No. 2, February 2011.

14. Archana Singh, AvantikaYadav and Ajay Rana," K-means with Three different Distance Metrics", International Journal of Computer Applications (0975 - 8887) Vol. 67, issue. 10, pp. 13-17, April 2013.

15. M. D. Malkauthekar," Analysis Of Euclidean Distance and Manhattan Distance Measure in Face Recognition", Third International Conference on Computational Intelligence an Information Technology (CIIT 2013), pp. 503-507, October 2013.

16. Chengjun Liu, “ Discriminant Analysis And Similarity Measure”, Elsevier Pattern Recognition, Vol.47, pp. 359-367, issue 1,January 2014.

17. B. Mak and E. Barnard, "Phone Clustering Using The Bhattacharyya Distance," International Conference on Spoken Language (ICSLP), vol. 4, pp. 2005-2008, 1996.

18. C. Sheth and R V. Babu, "Object Saliency Using a Background Prior”, IEEE ICASSP, pp. 1931-1935, 2016.

19. Z. Yu, P. Luo, J.e You, H.-S. Wong, H. Leung, S. Wu, J. Zhang, and G. Han, "Incremental Semi-Supervised Clustering Ensemble for High Dimensional Data Clustering”, IEEE Transactions on Knowledge and Data Engineering, vol. 28, no. 3, pp. 701-714, 2016.

20. Frank Nielsen," A Generalization of the Jensen Divergence: The Chord Gap Divergence" Machine Learning arxiv: 1709.10498v2, November 2017 (ICASSP 2 018).

21. Jun Fengand H. S. Ip Horace, " Chi-Square Goodness-of-Fit Test of 3D Point Correspondence for Model Similarity Measure and Analysis", International Conference on Image and Video Retrieval : Image and Video Retrieval, pp 445-453,August 2005.

22. AmandeepKhokher, Dr. Rajneesh Talwar, "Content Based Image Retrieval: State-of-the-Art and Challenges,” International Journal of Advanced Engineering Sciences and Technologies, Vol No. 9, Issue No. 2, 207-211

23. A.Kannan, Dr.V.Mohan, Dr.N.Anbazhagan, "An Effective Method of Image Retrieval using Image Mining Techniques," The International Journal of Multimedia \& Its Applications (IJMA) Vol.2, No.4, November 2010.

24. John R. Smith and Shih-Fu Chang, "VisualSEEk: a Fully Automated Content-Based Image Query System" ACM Multimedia 96, Boston, MA, November 20, 1996

25. E. Binaghi, I. Gagliardi, R. Schettini, "Image Retrieval Using Fuzzy Evaluation of Color Similarity", International Journal Of Pattern Recognition And Artificial Intelligence, Vol. 18, pp. 945$968,1994$.

26. B. Barolo, I. Gagliardi, R. Schettini, “An Effective Strategy for Querying Image Databases by Color Distribution Computer and the History of Art Journal, Vol.7(1), pp. 3-14,1997.

27. J. Hafner,H.S. Sawhney, M. Esquitz.W,Flickner, W. Niblack, "Efficient Color Histogram Indexing For Quadratic Form Distance Functions", IEEE Transactions on Pattern Analysis and Machine Intelligence, Vol. 17,pp. 729-736, 1995

28. M.S.KanKanhalli, B.M. Mehtre, H.Y.Huang, "Color and Spatial Feature for Content Based Information Retrieval", Pattern Recognition Letters, Vol.20, pp.109-118,1999.

29. J.R.Smith, and S.F.Chan," Single Color Extraction and Image Query", Proceeding ICIP 1995.

30. I.K.Park, I.D.Yun, S.U.Lee, " Color Image Retrieval Using Hybrid Graph Representation", Image and Vision Computing, Vol.17,pp.465-474,1999.

31. M.A.Stricker," Color And Geometry as Cues for Indexing", Technical Report,CS92-99,1992.

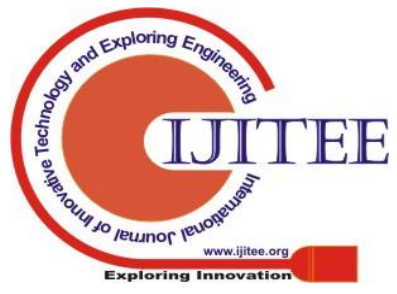


32. A.Vailaya, A.Jain,H.J.Zhang, " On Image Classification: Cite Images Vs. Landscape", Pattern Recognition, Vol.31, pp.19211935, 1998.

33. A.Vellaikal,C.C.JayKuo and S.Dao, " Content Based Retrieval Of Color and Multi Spectral Images Using Joint Spatial-Spectral Indexing", SPIE, Vol.2606,pp.232-243.

34. H.S.Sawhney, and J.L.Hafner, "Efficient Color Histogram Indexing",IEEE International Conference on Image Processing,vol.2,pp.66-70,1994.

35. Siddhartha Bhattacharyya, ParamarthaDutta, UjjwalMaulik and Prashanta Kumar Nandi, "Multilevel Activation Functions For True Color Image Segmentation Using a Self Supervised Parallel Self Organizing Neural Network (PSONN) Architecture: A Comparative Study" International Journal of Electrical and Computer Engineering 2:1,PP.9-21, 2007.

36. JyotiNarwade, Binod Kumar, "Local and Global Color Histogram Feature for Color Content-Based Image Retrieval System" ,Proceedings of the International Congress on Information and Communication Technology, ISC, volume 438) pp 293-300.

37. Y. Liu, D. Zhang, G. Lu, and W. Y. Ma, "A Survey of ContentBased Image Retrieval With High-Level Semantics", Pattern Recognition, Vol. 40, no. 1, pp. 262-282, 2007.

38. F. Long, H. Zhang, H. Dagan, and D. Feng, "Fundamentals of Content Based Image Retrieval," In Multimedia Information Retrieval and Management, D. Feng, W. Siu, and H. Zhang, Eds., Multimedia Signal Processing Book, chapter 1, pp. 1-26, Springer, Berlin, Germany, 2003.

39. Ahmed J. Afifi and Wesam M. Ashour, “ Image Retrieval Based on Content Using Color Feature”, International Scholarly Research NoticesComputer Graphics, Vol. 2012,pp.1-11.

40. M. Stricker and A.Dimai,'Spectral Covariance and Fuzzy Regions for Image Indexing Machine Vision And Applications",vol.10,pp.66-73,1997.

41. M. Stricker, and A.Dimai, "Color Indexing With Weak Spatial,Constraints" In Proceedings of SPIE Storage and Retrieval for Image and Video Databases, 1996, pp. 29-40.

\section{AUTHORS PROFILE}

A.Jeya Christy, Research Scholar, Department of Computer Science and Engineering, Vaigai College of Engineering, Madurai, Tamil Nadu, India.

Dr. K. Dhanalakshmi, Professor, Department of Computer Science and Engineering, PSNA College of Engineering and Technology, Dindigul, Tamil Nadu, India. 